\title{
Retrospective Analysis of Intravaginal Brachytherapy in Adjuvant Treatment of Early Endometrial Cancer
}

\author{
Pawel Cisek (D), ${ }^{1,2}$ Dariusz Kieszko, ${ }^{2}$ Izabela Kordzińska-Cisek, ${ }^{1}$ \\ Elżbieta Kutarska, ${ }^{3}$ and Ludmiła Grzybowska-Szatkowska ${ }^{1}$ \\ ${ }^{1}$ Department of Oncology, Medical University of Lublin, Jaczewskiego 7, Lublin, Poland \\ ${ }^{2}$ Department of Brachytherapy, St. John's Oncology Center of Lublin, Jaczewskiego 7, Lublin, Poland \\ ${ }^{3}$ Department of Gynecology, St. John's Oncology Center of Lublin, Jaczewskiego 7, Lublin, Poland \\ Correspondence should be addressed to Paweł Cisek; pcisek@interia.eu
}

Received 30 August 2017; Revised 20 December 2017; Accepted 28 December 2017; Published 21 February 2018

Academic Editor: John P. Geisler

Copyright (C) 2018 Paweł Cisek et al. This is an open access article distributed under the Creative Commons Attribution License, which permits unrestricted use, distribution, and reproduction in any medium, provided the original work is properly cited.

\begin{abstract}
The aim of this study was to determine the role of adjuvant endovaginal brachytherapy HDR (High Dose Rate) or observation, as well as identification of risk factors of tumor recurrence. The study included 178 women after radical hysterectomy. All patients belonged to the group of low- and medium-risk stage I FIGO. Analysis consisted of 3-, 5-, and 10-year OS, DFS, and LRFS in both groups. Follow-up was more than 6.5 years. The 5-OS, 5-DFS, and 5-LRFS were $93 \%, 96 \%$, and $98 \%$ in the treated group and $95 \%, 94 \%$, and $96 \%$ in the observed group, respectively. These differences were not statistically significant. There was a statistically significant difference in 5-OS in the treated group, between low- and medium-risk subgroups (100\% versus $87.55 \%, p=0.018$ ). There was a better prognosis among the patients with FIGO IA compared to FIGO IB (5-DFS, 97 versus 86\%, $p=0.047$ ). Among the risk factors, there were only statistically significant differences in the 5-OS, between the ages of $\leq 70$ years and $>70$ years. Use of brachytherapy may affect the reduction in the number of local recurrences at the vaginal stump (6\% versus $2 \%$ ). This is particularly noticeable in the low-risk subgroup ( $9 \%$ versus $0 \%$ ).
\end{abstract}

\section{Introduction}

Uterine cancer is the sixth most common cancer in women in the world and the fourth most common cancer in Europe. It is also the second most common cancer among gynecological cancers after cervical cancer in the world and first in Europe [1]. The management of endometrial cancer is complex and it is based on surgical treatment, radiotherapy, chemotherapy, and hormonal therapy. A recommended and routine surgical procedure is the total abdominal hysterectomy with bilateral salpingooophorectomy [2]. The role of pelvic lymphadenectomy has not been definitively established and remains controversial [3]. Depending on the stage of cancer, exclusive observation, teleradiotherapy, brachytherapy, a combination therapy involving both of these methods, or chemotherapy are used after surgery [2].

In general, no supplementary treatment [2] is used in the low-risk group (IA, G1, G2, and endometrioid type).
Literature analysis indicates no benefit in overall survival from complementary radiotherapy [4-8]. There are also no new, large randomized trials comparing complementary brachytherapy with exclusive observation in this group of patients. The decision regarding complementary treatment or follow-up in other patients with FIGO (International Federation of Gynecology and Obstetrics) IA is usually based on the presence of a number of factors, which are considered unfavorable prognosis. These include the following: age > 70 years, histopathological type II or mixed (according to Bokhman), low tumor descent, tumor mass $>2 \mathrm{~cm}$, low uterine segment involvement (LUSI), and histopathological grade G3 [2]. There is even more controversy about patients with FIGO IB-there are no studies comparing exclusive surveillance with independent brachytherapy in this group of patients. Treatment usually depends on the presence of risk factors, the hospital's own experience, and the patient's preference. In this study, we performed a 


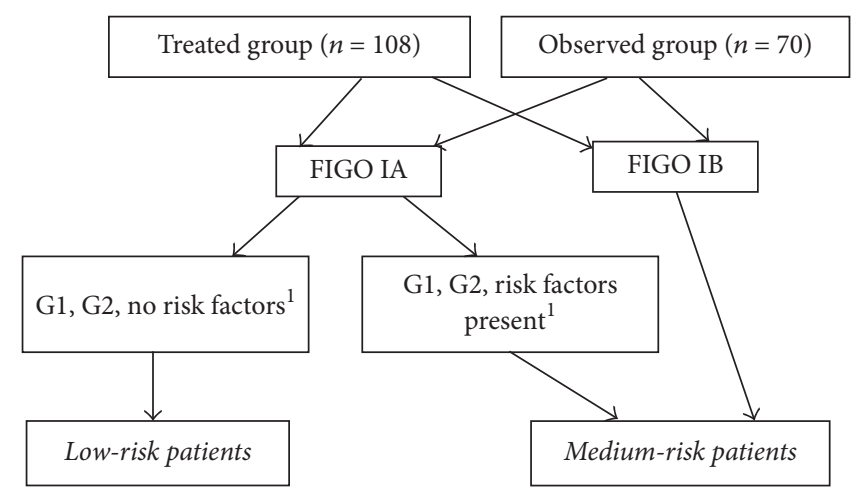

FIGURE 1: Division into prognosis subgroups. ${ }^{1}$ Risk factors of recurrence: histopathological type II or mixed, low tumor descent, tumor size $>2 \mathrm{~cm}$, age $>70$ years, and degree of malignancy $\mathrm{G} 3$.

one-sided, comparative retrospective analysis of patients with early-stage endometrial cancer treated with complementary brachytherapy or postoperative follow-up.

\section{Material and Methods}

2.1. Characteristics of Patients. The retrospective analysis included 178 patients with histopathologically confirmed invasive endometrial cancer in the first stage of FIGO clinical stage, treated by brachytherapy or subjected to a control study in 1989-2013. All patients were after total abdominal hysterectomy with bilateral salpingooophorectomy. Node dissection was optional. All patients were treated with a radical premise. The patients were divided according to the scheme shown in Figure 1 into two groups:

(1) Treated group: who received adjuvant brachytherapy

(2) Observed group: who used only observation.

Then, depending on clinical and histopathological factors, the two groups were divided according to the scheme shown in Figure 1 into patients with low and medium risk.

There were 49 low-risk and 59 medium-risk patients in the treated group and 32 low-risk patients and 38 mediumrisk patients in the observed group (Table 1).

2.2. Treatment. Patients from both groups had undergone uterine abduction with pelvic lymphadenectomy or no lymphadenectomy. Patients in the treated group were irradiated by HDR (High Dose Rate) brachytherapy using Ir192 source. The treatment was started from the placement of a singletube cylindrical applicator into the vagina and an X-ray examination to plan the treatment. Treatment planning was to determine the source stops and stopping time of the source in the applicator to cover with the prescribed isodose the upper $1 / 3$ part of the vaginal mucosa at a depth of $5 \mathrm{~mm}$. The dose was also calculated at the ICRU (International Commission on Radiation Units and Measurements) of the bladder and rectum and the maximum dose in the vaginal mucosa. Median fractional dose was 7.5 (6-8) Gy and total dose 30 (15-32) Gy. Most 3-4 fractions were used at weekly intervals.
TABLE 1: Characteristics of patients.

\begin{tabular}{lcc}
\hline $\begin{array}{l}\text { Demographical or clinical } \\
\text { risk factor }\end{array}$ & $\begin{array}{c}\text { Treated group, } \\
n=108\end{array}$ & $\begin{array}{c}\text { Observed group } \\
n=70\end{array}$ \\
\hline $\begin{array}{l}\text { Age of patients: median } \\
\text { (range) }\end{array}$ & $65(47-90)$ years & $66(46-90)$ years \\
FIGO: & $92(85 \%)$ & $59(84 \%)$ \\
IA & $16(15 \%)$ & $11(16 \%)$ \\
IB & & \\
Number of risk factors & $53(49 \%)$ & $35(50 \%)$ \\
0 & $51(47 \%)$ & $29(41 \%)$ \\
1 & $4(4 \%)$ & $6(9 \%)$ \\
2 & & $2(3 \%)$ \\
Type of risk factors & $9(8 \%)$ & $2(3 \%)$ \\
LUSI & $11(10 \%)$ & $13(19 \%)$ \\
Size of tumor $>2 \mathrm{~cm}$ & $4(4 \%)$ & $9(9 \%)$ \\
Histopathologic type II & $2(2 \%)$ & $23(33 \%)$ \\
G3 & $37(34 \%)$ & \\
Age $>70$ lat &
\end{tabular}

LUSI: lower uterine segment involvement.

Patients undergoing exclusive follow-up and postbrachytherapy patients were assigned for a follow-up visit every three months during the first two years and then every six months and every five years. The primary outcome measure was the 3-, 5-, and 10-year OS, DFS, and LRFS rates, depending on the type of follow-up and prognosis. The second outcome was identification of risk factors of tumor recurrence. Statistical analysis was performed with the Kaplan-Meier method along with the log-rank test.

\section{Results}

3.1. Overall Survival (OS). Patients included in the study were followed for an average of $67,46 \pm 45,27$ months after treatment (over 6.5 years). In the treated group, the mean follow-up was $48.74 \pm 20.15$ months (over 4 years). In the observed group the mean follow-up was longer, $96.35 \pm 56.86$ months ( 8 years). The median follow-up time in both groups was 58.88 months 
TABLE 2: 3-, 5-, and 10-OS, DFS, and LRFS in treated and observed groups and among patients with low and medium risk of recurrence.

\begin{tabular}{|c|c|c|c|c|}
\hline \multicolumn{5}{|c|}{ Groups and subgroups } \\
\hline & 3-OS & $5-\mathrm{OS}$ & $10-\mathrm{OS}$ & \\
\hline Treated & $96,86 \%$ & $93 \%$, & $81,8 \%$ & \multirow{3}{*}{ NS } \\
\hline versus & & & & \\
\hline Observed & $100 \%$ & $95,12 \%$ & $93,3 \%$ & \\
\hline \multicolumn{5}{|l|}{ Treated } \\
\hline Low risk & $100 \%$ & $100 \%$ & $93,75 \%$ & \multirow{3}{*}{$\begin{array}{c}\text { SS } \\
p=0.018\end{array}$} \\
\hline versus & & & & \\
\hline Medium risk & $94,34 \%$ & $87,55 \%$ & $72,01 \%$ & \\
\hline \multicolumn{5}{|l|}{ Observed } \\
\hline Low risk & $100 \%$ & $100 \%$ & $96,15 \%$ & \multirow{3}{*}{ NS } \\
\hline versus & & & & \\
\hline \multirow[t]{2}{*}{ Medium risk } & $100 \%$ & $90,95 \%$ & $90,95 \%$ & \\
\hline & 3 - DFS & 5 -DFS & 10 - DFS & \multirow{4}{*}{ NS } \\
\hline Treated & $96,74 \%$ & $95,58 \%$ & $95,58 \%$ & \\
\hline versus & & & & \\
\hline Observed & $97,14 \%$ & $93,98 \%$ & $89,7 \%$. & \\
\hline \multicolumn{5}{|l|}{ Treated } \\
\hline Low risk & $100 \%$ & $97,5 \%$ & $97,5 \%$ & \multirow{3}{*}{ NS } \\
\hline versus & & & & \\
\hline Medium risk & $93,91 \%$ & $93,91 \%$ & $93,91 \%$ & \\
\hline \multicolumn{5}{|l|}{ Observed } \\
\hline Low risk & $93,75 \%$ & $93,75 \%$ & $84,38 \%$ & \multirow{3}{*}{ NS } \\
\hline versus & & & & \\
\hline \multirow[t]{2}{*}{ Medium risk } & $100 \%$ & $94,02 \%$ & $94,02 \%$ & \\
\hline & 3 - LRFS & 5 -LRFS & 10 - LRFS & \\
\hline Treated & $97,77 \%$ & $97,77 \%$ & $97,77 \%$, & \multirow{3}{*}{ NS } \\
\hline versus & & & & \\
\hline Observed & $97,14 \%$, & $95,57 \%$ & $91,75 \%$. & \\
\hline \multicolumn{5}{|l|}{ Treated } \\
\hline Low risk & $100 \%$ & $100 \%$ & $100 \%$ & \multirow{3}{*}{ NS } \\
\hline versus & & & & \\
\hline Medium risk & $96 \%$ & $96 \%$ & $96 \%$ & \\
\hline \multicolumn{5}{|l|}{ Observed } \\
\hline Low risk & $93,75 \%$ & $93,75 \%$ & $84,38 \%$, & \multirow{3}{*}{ NS } \\
\hline versus & & & & \\
\hline Medium risk & $100 \%$ & $97,06 \%$ & $97,06 \%$ & \\
\hline
\end{tabular}

OS: overall survival, DFS: disease-free survival, LRFS: local relapse-free survival.

(range 0-316.93 months), with 54.52 months (range 0-90.13) in the treated group and 87.22 months in the observed group (25.63-316,93 months).

In the treated group of 108 patients $9.3 \%$ (10/108) died during the observation period. In the observed group of 70 patients, 7.1\% (5/70) died during the observation period.

There were no statistically significant differences in overall survival between the treated and the observed group $(p>0.05)$ (Figure 2). In the treated group, there was a statistically significant difference in overall survival between the low- and the medium-risk patients $(p=0.018)$. There was no statistically significant difference between low risk and medium risk in the observed group $(p>0.05)$ (Figure 3$)$. In addition, among the low-risk and medium-risk patients, no statistically significant differences in overall survival between the treated and observed group $(p>0.05)$ were reported; $3-$, 5 -, and 10-year OS in groups and subgroups are shown in Table 2.

3.2. Disease-Free Survival (DFS). In the treated group, 4 patients $(4 \%)$ were documented for recurrence during the follow-up period. The median time to recurrence was 


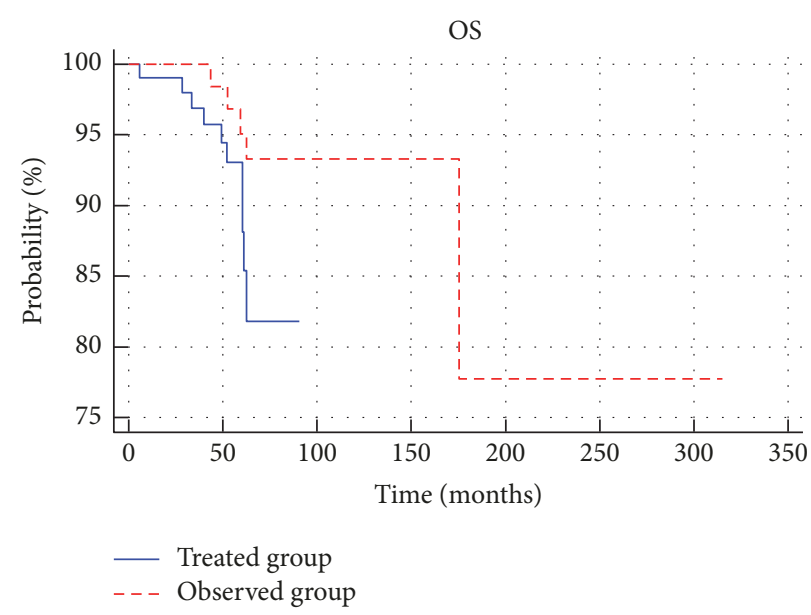

FIGURE 2: Comparison of overall survival between treated group and observed group.

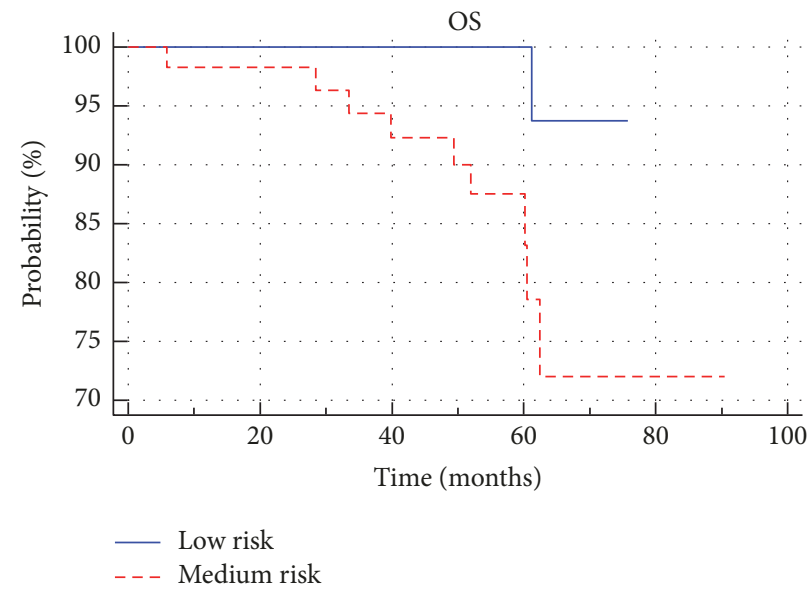

(a)

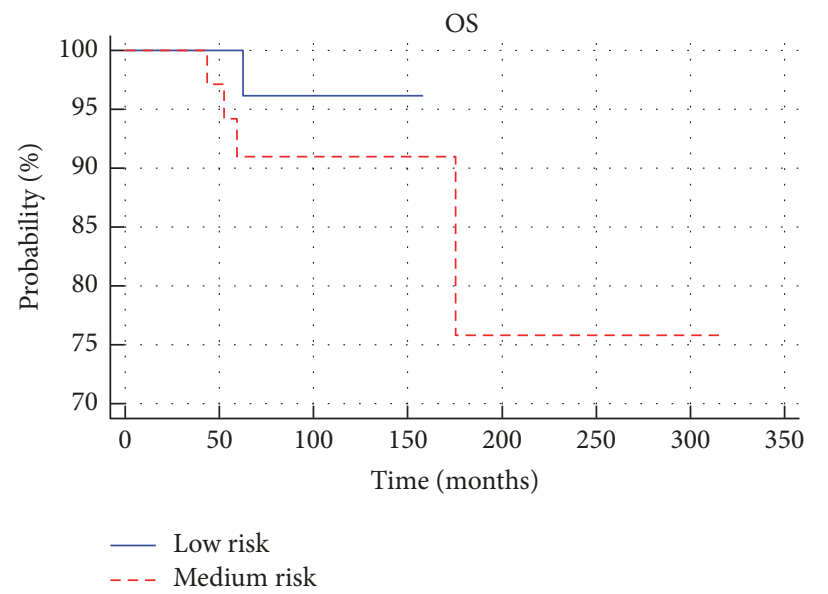

(b)

FIGURE 3: Comparison of overall survival between low- and medium-risk subgroups in the treated (a) and observed groups (b).

$47.54 \pm 20.91$ months, the median time to recurrence was 53.37 months (range 0-90 months). Two patients (2\%) had a recurrence at the vaginal stump area, and in the remaining 2 patients there were distant metastases to the lung and lymph nodes of the abdominal cavity. Both local recurrences occurred among medium-risk patients. Distant metastases were documented among patients with low and medium risk. In the observed group, the mean disease-free survival was $95.4 \pm 58.05$ with the median 87.77 months (range 13-320 months). During the whole period of observation a recurrence of the tumor was observed in 5 patients $(7 \%)$, in $4(6 \%)$ it was a local recurrence, and in 1 patient $(1 \%)$ it was lung metastases. Three patients with localized recurrence were among the low-risk patients, one among the mediumrisk patients. A distant recurrence was documented in the medium-risk group.

There were no statistically significant differences in disease-free survival between the treated group and the observed group $(p>0.05)$ (Figure 4$)$. No significant difference in disease-free survival between patients with low and medium risk $(p>0.05)$ was observed in either group (Figure 5). Among the low-risk and medium-risk patients, no statistically significant differences in disease-free survival between the treated and observed group $(p>0.05)$ were reported. Three-, 5- and 10-year-old DFS in the groups and the subgroups are presented in Table 2.

3.3. Local Relapse-Free Survival (LRFS). Local recurrences occurred in 2 patients (2\%) in the treated group and $4(6 \%)$ in the observed group $(p>0.05)$. In patients with a low risk of local recurrence none occurred in the treated group and 9\% occurred in the observed group. Among the medium-risk patients, the percentage of local recurrences in both groups was similar (3\%). There were no statistically significant differences in LRFS between patients in the treated group and the observed group $(p>0.05)$ (Figure 6). None of the groups showed statistically significant differences in the local relapse-free survival between low- and medium-risk patients (Figure 7). In addition, among the low-risk and mediumrisk patients, no statistically significant differences in local 


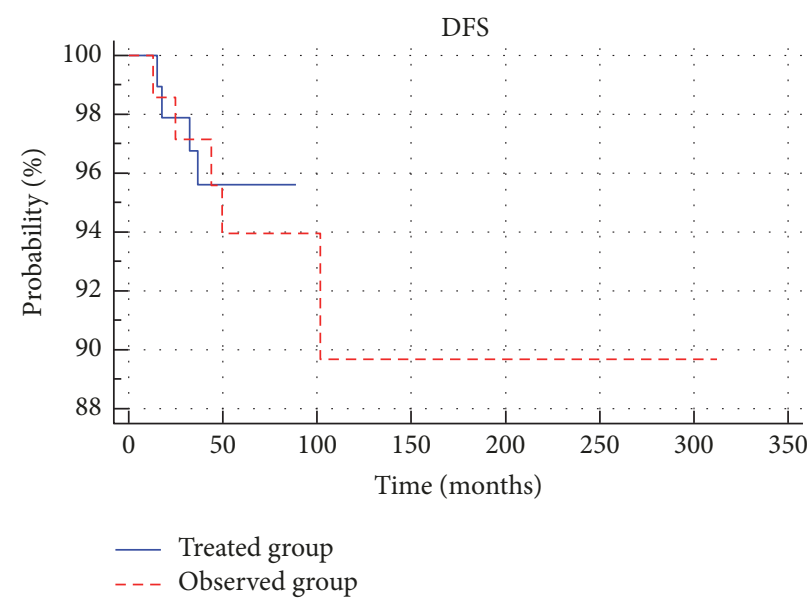

FIGURE 4: Comparison of disease-free survival between treated group and observed group.

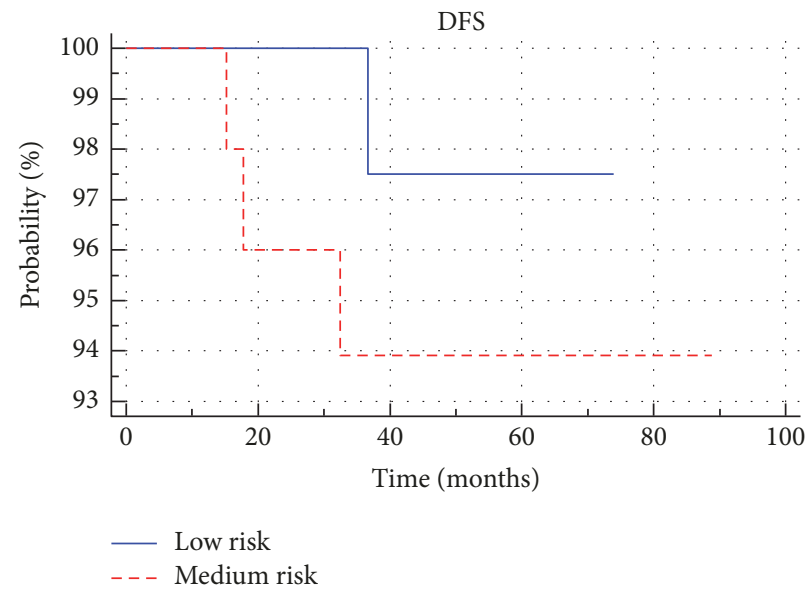

(a)

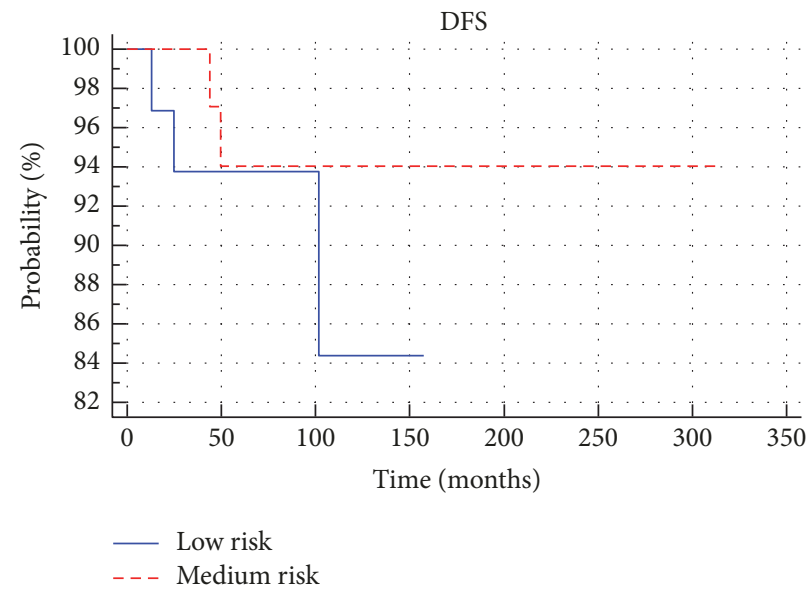

(b)

FIgURE 5: Comparison of disease-free survival between low- and medium-risk subgroups in the treated (a) and observed groups (b).

relapse-free survival between the treated and observed group ( $p>0.05$ ) were reported; 3-, 5-, and 10-year-old LRFS in groups and subgroups are shown in Table 2.

3.4. Impact of Risk Factors. In the treated and observed groups the influence of particular factors on the prognosis was analyzed. There was a statistically significant effect of age $(<70)$ on OS and the clinical stage on DFS. Other factors alone did not affect survival $(p>0.05)$. The results are presented in Table 3.

\section{Discussion}

Literature analysis in the low-risk group (Ia-G1, G2, and endometrioid type) indicates no benefit in overall survival from complementary brachytherapy [2]. In many cases, exclusive observation in early endometrial cancer seems to be sufficient. Very good results were obtained using also brachytherapy $[12,19,26]$. A comparison of exclusive observation and brachytherapy conducted by Sorbe et al. [9] as well as the analysis of own material indicates no statistically significant difference in the recurrence rate.

Controversy raises the value of complementary treatment in the case of FIGO IB or FIGO IA, which coincides with age-related factors such as age $>70$ years, histopathological type II or mixed (according to Bokhman), low tumor descent, tumor size $>2 \mathrm{~cm}$, LVSI, and histopathological grade G3. Depending on the studies in which they were evaluated and the risk factors for which they were classified in the prognostic groups, patients with recurrence risk factors were usually classified as either medium or high risk [4-6]. In the analyzed study, the medium-risk group consisted of FIGO IA patients with risk factors for recurrence (age $>70$ years, histopathological type II or mixed, low tumor descent, tumor size $>2 \mathrm{~cm}$, and histopathological grade G3) or patients with FIGO IB, irrespective of the presence of other factors.

Studies show that in this group of patients after the use of adjuvant brachytherapy the rate of recurrence in the vagina is very low and the rate of survival free from recurrence and overall survival is high, even when the total dose is lower 
TABLE 3: 5-OS and 5-DFS in patients with risk factors.

\begin{tabular}{|c|c|c|c|c|c|c|c|c|}
\hline & \multicolumn{4}{|c|}{$5-\mathrm{OS}$} & \multicolumn{4}{|c|}{ 5-DFS } \\
\hline & Treated group & $p$ & Observed group & $p$ & Treated group & $p$ & Observed group & $p$ \\
\hline FIGO IA & $88,71 \%$ & NS & $96,11 \%$, & NS & $97,35 \%$ & 0.047 & $92,92 \%$ & NS \\
\hline FIGO IB & $84,41 \%$ & 10 & $90,00 \%$ & 10 & $85,71 \%$ & 0.013 & $100 \%$ & \\
\hline $\begin{array}{l}\text { No risk } \\
\text { factors }\end{array}$ & $95,65 \%$ & & $100 \%$ & & $100 \%$ & & $100 \%$ & \\
\hline 1 risk factor & $81,59 \%$ & 0.015 & $88,14 \%$ & NS & $97,72 \%$ & NS & $92,14 \%$ & NS \\
\hline 2 risk factors & $66,66 \%$ & & $100 \%$ & & $92,96 \%$ & & $94,28 \%$ & \\
\hline Age $>70$ & $92,86 \%$ & 0.011 & $95,29 \%$ & NS & $98,27 \%$ & NS & $93,35 \%$ & NS \\
\hline Age $<70$ & $77,81 \%$ & & $94,44 \%$ & & $89,87 \%$ & $p=0.1$ & $95,23 \%$ & \\
\hline
\end{tabular}

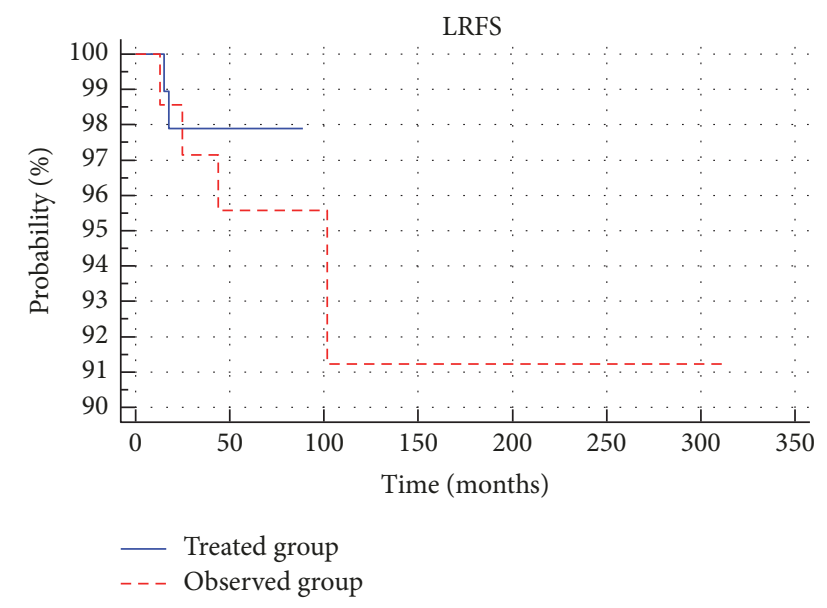

FIGURE 6: Comparison of local relapse-free survival between treated group and observed group.

than our analysis $[10,11,13-15,17,18,20-25]$ (Table 4). There are no large trials comparing independent brachytherapy and observation in the medium-risk group or high-intermediaterisk group. Nevertheless, PORTEC has shown that $72 \%$ of recurrences occur in the vagina, and, in GOG-99, 77.8\% of all failures occurred in the vagina [4-6]. The analysis of own material in the medium-risk group indicates the lack of benefits from adjuvant brachytherapy in OS, DFS, and LRFS.

Analysis of all groups confirms the possible effect of brachytherapy on the reduction of local recurrence rate $(6 \%$ in the observed group versus $2 \%$ in the treated group, NS). This is particularly evident among low-risk patients ( $9 \%$ in the observed group versus $0 \%$ in the treated group, NS). Unfortunately, the relapse rate in this study was too low to obtain statistical significance.

Many studies indicate deterioration of prognosis with the depth of uterine muscle invasion. Dunn et al. [27] in Cox's univariate analysis indicate that the degree of infiltration of muscle is an independent risk factor for a deterioration of the prognosis $(p=0.004)$. Some authors diminish the role of the depth of infiltration. Aristizabal et al. [28] did not show differences in 5-OS between FIGO IA and IB patients. In the analyzed group only the treated group showed a deterioration of relapse-free survival without effect on overall survival $(p=0.047)$. Among the other factors, only the elderly were affected by a deterioration of survival, which is understandable because of the likely higher incidence of coexisting diseases in this age group. The effects of advanced age on the percentage of relapses were not reported. Studies show that older age favors deterioration of overall survival by $12 \%$, relapse-free survival by $11 \%$, and local recurrence by $8 \%$ $[27,29]$. Similarly in the study of Arenas et al. [30] age $<75$ years and myometrial invasion $\leq 50 \%$ are predictors of a good outcome in endometrial cancer.

Many studies indicate an effect on prognosis of other risk factors such as tumor localization, tumor size $>2 \mathrm{~cm}$, Bokhman tumor type II, or G3 grade [28, 30-33]. While the effect of G3 grade or some type of histopathologic type II tumor type does not leave room for doubt, the role of tumor size or location is questionable among many researchers [34, 35]. No statistically significant effects of the above described risk factors on OS or DFS were reported in the study group. These results may, however, be associated with a small number of patients with particular risk factors and a low number of recurrences and deaths in the analyzed group of patients.

The limitation of the study was a retrospective analysis of patients. This analysis included more than 10 years of follow-up, during which indications for adjuvant therapy were evolved to reduce brachytherapy. This causes an unequal distribution of some risk factors in both groups; however, a small percentage of them does not seem to affect the final outcomes. Another reason was the tendency to select patients depending on the type of risk factors like LUSI, size of tumor, and histopathologic type and grade.

\section{Conclusion}

Among patients with early endometrial cancer the prognosis is good, regardless of treatment administered. Brachytherapy effects on OS, DFS, and LRFS have not been demonstrated. Based on the above data, patients from the low- and intermediate-risk groups should be observed after surgical treatment. The use of brachytherapy in the low-risk group (FIGO IA, G1, 2, and no risk factors) may reduce the incidence of localized recurrences in the vagina although, due to the small percentage of relapses, these differences are not statistically significant. In the medium-risk group (IB 
TABLE 4: Fractionation schemes and survival parameters.

\begin{tabular}{|c|c|c|c|c|}
\hline Author & Fractional dose & Total dose & Survival parameters & Follow-up \\
\hline Sorbe et al. [9] & $3-8 \mathrm{~Gy} / 5 \mathrm{~mm}$ & $18-24$ Gy & $\begin{array}{l}\text { LRR: } 2,6 \%, \text { DM: } 1,3 \% \text {, } \\
\text { VR: } 1,2 \%\end{array}$ & - \\
\hline Weiss et al. [10] & $\begin{array}{l}4,6-4,9 \mathrm{~Gy} / 5 \mathrm{~mm} \\
(7 \mathrm{~Gy} / \text { surface })\end{array}$ & 13,8-14,7 Gy & $\begin{array}{c}\text { Pelvic relapse } 5,7 \% \text {, vaginal stump relapse } 1,6 \% \text {, local } \\
\text { and distance relapse } 2,5 \%, \mathrm{DM}: 1,6 \%, 5 \text {-RFS } 74 \% \text { in MR } \\
\text { group and } 94 \% \text { in HR group }\end{array}$ & $25,6 \mathrm{~m}$ \\
\hline Chadha et al. [11] & $7 \mathrm{~Gy} / 5 \mathrm{~mm}$ & $21 \mathrm{~Gy}$ & VR: $0 \%, 5$-OS: 93\%, 5-DFS: $87 \%$ & $30 \mathrm{~m}$ \\
\hline Anderson et al. [12] & $5 \mathrm{~Gy} / 5 \mathrm{~mm}$ & $15 \mathrm{~Gy}$ & $\begin{array}{l}\text { Pelvic relapse } 3 \% \text {, vaginal relapse } 1 \%, 5 \text {-OS } 84 \%, 5 \text {-DFS } \\
93 \% \text {, }\end{array}$ & - \\
\hline Alektiar et al. [13] & $6-7 \mathrm{~Gy} / 5 \mathrm{~mm}$ & $18-21 \mathrm{~Gy}$ & 5-OS 93\%, 5-DFS 97\% & $48 \mathrm{~m}$ \\
\hline $\begin{array}{l}\text { McCloskey et al. } \\
{[14]}\end{array}$ & $7 \mathrm{~Gy} / 5 \mathrm{~mm}$ & $21 \mathrm{~Gy}$ & $\begin{array}{c}\text { Local relapse } 3,4 \% \text {, vaginal relapse } 1,1 \% \text {, vaginal and } \\
\text { pelvic relapse } 1,1 \% \text {, pelvic relapse } 1,1 \%\end{array}$ & $52 \mathrm{~m}$ \\
\hline Ríos et al. [15] & $4 \mathrm{~Gy} / 5 \mathrm{~mm}$ & 20 Gy & $\begin{array}{c}\text { Vaginal stump relapse } 0 \% \text {, relapse in lower part of } \\
\text { vagina } 1,7 \%, \mathrm{DM} 6,7 \%\end{array}$ & $46,7 \mathrm{~m}$ \\
\hline PORTEC-2 [16] & $7 \mathrm{~Gy} / 5 \mathrm{~mm}$ & $21 \mathrm{~Gy}$ & 5-VR: $1,8 \%, 5$-LRR: 5,1\%, 5-OS 84,8\%, 5-DFS $82,7 \%$ & $45 \mathrm{~m}$ \\
\hline Lin et al. [17] & $7 \mathrm{~Gy} / 5 \mathrm{~mm}$ & $21 \mathrm{~Gy}$ & 5-OS $86 \%, 5$-DFS $89 \%$ & $55 \mathrm{~m}$ \\
\hline Atahan et al. [18] & $5,5 \mathrm{~Gy} / 5 \mathrm{~mm}$ & $27,5 \mathrm{~Gy}$ & VR: $1,6 \%$, DM 3,2\%, 5-OS 96\%, 5-DFS 93\% & $48 \mathrm{~m}$ \\
\hline Solhjem et al. [19] & $7 \mathrm{~Gy} / 5 \mathrm{~mm}$ & $21 \mathrm{~Gy}$ & No local relapses & $23 \mathrm{~m}$ \\
\hline Cengiz et al. [20] & $7 \mathrm{~Gy} / 5 \mathrm{~mm}$ & $21 \mathrm{~Gy}$ & 5-OS 85\%, 5-DFS 92\%, 5-LC 95\% & $54 \mathrm{~m}$ \\
\hline $\begin{array}{l}\text { Rittenberg et al. } \\
{[21]}\end{array}$ & $5,6 \mathrm{~Gy} / 5 \mathrm{~mm}$ & $16,8 \mathrm{~Gy}$ & VR: 2,3\% 2-OS 97\%, 5-OS 95\% & $32 \mathrm{~m}$ \\
\hline Horowitz et al. [22] & $7 \mathrm{~Gy} / 5 \mathrm{~mm}$ & $21 \mathrm{~Gy}$ & $\begin{array}{l}\text { All relapse: } 8,5 \% \text {, vaginal relapses: } 2 \%, 5 \text {-OS } 87 \% \text {, } \\
5 \text {-DFS } 90 \%\end{array}$ & $65 \mathrm{~m}$ \\
\hline Rovirosa et al. [23] & $5-6 \mathrm{~Gy} / 5 \mathrm{~mm}$ & $20-24$ Gy & No vaginal relapses & $75 \mathrm{~m}$ \\
\hline Rovirosa et al. [23] & $4-6 \mathrm{~Gy} / 5 \mathrm{~mm}$ & $24-36 \mathrm{~Gy}$ & No vaginal relapses & $88 \mathrm{~m}$ \\
\hline Rovirosa et al. [24] & $6 \mathrm{~Gy} / 5 \mathrm{~mm}$ & 18 Gy & No vaginal relapses & $41 \mathrm{~m}$ \\
\hline $\begin{array}{l}\text { Townamchai et al. } \\
\text { [25] }\end{array}$ & 4 Gy/surface & 24 Gy & $\begin{array}{l}\text { Vaginal relapses } 1,2 \% \text {, para-aortic nodes relapses } 1,9 \% \text {, } \\
\text { DM } 1,2 \%\end{array}$ & $22,8 \mathrm{~m}$ \\
\hline
\end{tabular}

LRR: locoregional relapses, DM: distal metastases, VR: vaginal recurrence, RFS: recurrence-free survival, MR: mediate risk, HR: high risk, m: months.

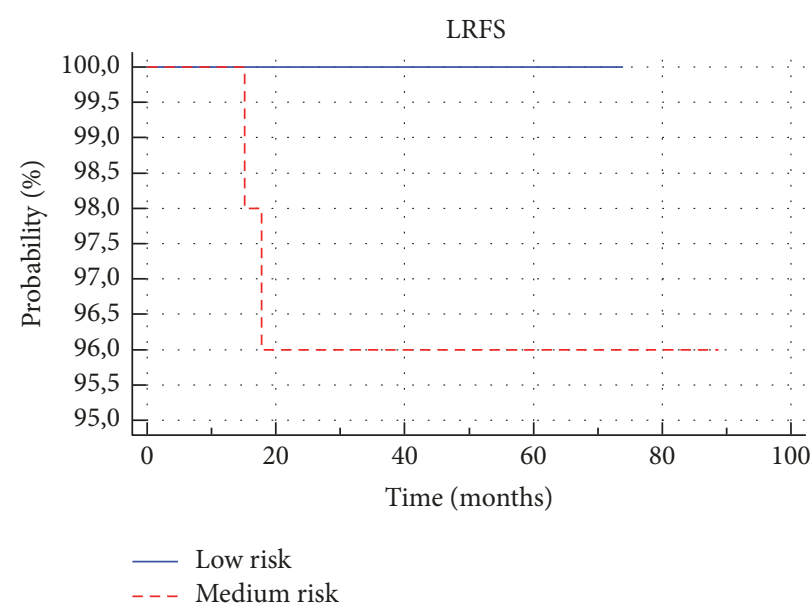

(a)

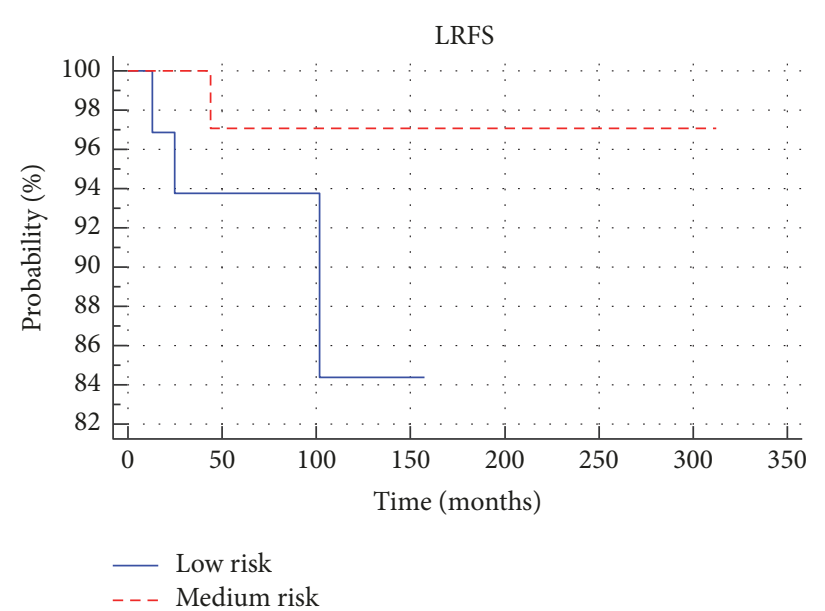

(b)

FIGURE 7: Comparison of local relapse-free survival between low- and medium-risk subgroups in the treated (a) and observed groups (b).

and/or risk factors) there was no reduction in the rate of relapse after brachytherapy. Because of the lower OS in the medium-risk group, compared to the low-risk group, further research is needed to find new treatments that will improve the outcome.
The clinical stage of FIGO IB increases the risk of recurrence, although it has no effect on survival. The older age deteriorates overall survival, without affecting diseasefree survival. Factors such as tumor size, low tumor location, G3, or Bokhman type II do not affect prognosis, although due 
to the low rate of relapses and deaths, as well as the small number of patients with particular factors, this issue requires further investigation on a larger group of patients.

\section{Conflicts of Interest}

The authors declare that there are no conflicts of interest regarding the publication of this article.

\section{References}

[1] GLOBOCAN: Cancer Incidence and Mortality Worldwide. Lyon: IARC, http://globocan.iarc.fr 2012.

[2] S. Sabater, I. Andres, V. Lopez-Honrubia et al., "Vaginal cuff brachytherapy in endometrial cancer - a technically easy treatment?" Cancer Management and Research, vol. 9, pp. 351-362, 2017.

[3] A. Mariani, S. C. Dowdy, W. A. Cliby et al., "Prospective assessment of lymphatic dissemination in endometrial cancer: a paradigm shift in surgical staging," Gynecologic Oncology, vol. 109, no. 1, pp. 11-18, 2008.

[4] H. M. Keys, J. A. Roberts, V. L. Brunetto et al., "A phase III trial of surgery with or without adjunctive external pelvic radiation therapy in intermediate risk endometrial adenocarcinoma: a Gynecologic Oncology Group study,' Gynecologic Oncology, vol. 92, no. 3, pp. 744-751, 2004.

[5] C. L. Creutzberg, W. L. van Putten, P. C. Koper et al., "Surgery and postoperative radiotherapy versus surgery alone for patients with stage-1 endometrial carcinoma: multicentre randomised trial," The Lancet, vol. 355, no. 9213, pp. 1404-1411, 2000.

[6] P. Blake, A. M. Swart, J. Orton et al., "Adjuvant external beam radiotherapy in the treatment of endometrial cancer (MRC ASTEC and NCIC CTG EN.5 randomised trials): pooled trial results, systematic review, and meta-analysis," The Lancet, vol. 373, no. 9658, pp. 137-146, 2009.

[7] N. Johnson and P. Cornes, "Survival and recurrent disease after postoperative radiotherapy for early endometrial cancer: Systematic review and meta-analysis," BJOG: An International Journal of Obstetrics \& Gynaecology, vol. 114, no. 11, pp. 13131320, 2007.

[8] A. Kong, I. Simera, M. Collingwood, C. Williams, and H. Kitchener, "Adjuvant radiotherapy for stage I endometrial cancer: Systematic review and meta-analysis," Annals of Oncology, vol. 18, no. 10, pp. 1595-1604, 2007.

[9] B. Sorbe, B. Nordström, J. Mäenpää et al., "Intravaginal brachytherapy in FIGO stage I low-risk endometrial cancer: A controlled randomized study," International Journal of Gynecological Cancer, vol. 19, no. 5, pp. 873-878, 2009.

[10] E. Weiss, P. Hirnle, H. Arnold-Bofinger, C. F. Hess, and M. Bamberg, "Adjuvant vaginal high-dose-rate afterloading alone in endometrial carcinoma: Patterns of relapse and side effects following low-dose therapy," Gynecologic Oncology, vol. 71, no. 1, pp. 72-76, 1998.

[11] M. Chadha, P. J. Nanavati, P. Liu, J. Fanning, and A. Jacobs, "Patterns of failure in endometrial carcinoma stage IB grade 3 and IC patients treated with postoperative vaginal vault brachytherapy," Gynecologic Oncology, vol. 75, no. 1, pp. 103-107, 1999.

[12] J. M. Anderson, B. Stea, A. V. Hallum, E. Rogoff, and J. Childers, "High-dose-rate postoperative vaginal cuff irradiation alone for stage IB and IC endometrial cancer," International Journal of Radiation Oncology Biology Physics, vol. 46, no. 2, pp. 417-425, 2000.

[13] K. M. Alektiar, E. Venkatraman, D. S. Chi, and R. R. Barakat, "Intravaginal brachytherapy alone for intermediaterisk endometrial cancer," International Journal of Radiation Oncology Biology Physics, vol. 62, no. 1, pp. 111-117, 2005.

[14] S. A. McCloskey, N. E. Tchabo, H. K. Malhotra et al., "Adjuvant vaginal brachytherapy alone for high risk localized endometrial cancer as defined by the three major randomized trials of adjuvant pelvic radiation," Gynecologic Oncology, vol. 116, no. 3, pp. 404-407, 2010.

[15] I. Ríos, A. Rovirosa, C. Ascaso et al., "Vaginal-cuff control and toxicity results of a daily HDR brachytherapy schedule in endometrial cancer patients," Clinical and Translational Oncology, vol. 18, no. 9, pp. 925-930, 2016.

[16] R. A. Nout, "Vaginal brachytherapy versus external beam pelvic radiotherapy for high-intermediate risk endometrial cancer: Results of the randomized PORTEC-2 trial," Clinical Oncology, vol. 26, no. Supplement abstr LBA5503, 2008.

[17] L. L. Lin, D. G. Mutch, J. S. Rader, M. A. Powell, and P. W. Grigsby, "External radiotherapy versus vaginal brachytherapy for patients with intermediate risk endometrial cancer," Gynecologic Oncology, vol. 106, no. 1, pp. 215-220, 2007.

[18] I. L. Atahan, E. Ozyar, F. Yildiz et al., "Vaginal high dose rate brachytherapy alone in patients with intermediate- to high-risk stage I endometrial carcinoma after radical surgery," International Journal of Gynecological Cancer, vol. 18, no. 6, pp. 1294-1299, 2008.

[19] M. C. Solhjem, I. A. Petersen, and M. G. Haddock, "Vaginal brachytherapy alone is sufficient adjuvant treatment of surgical stage I endometrial cancer," International Journal of Radiation Oncology Biology Physics, vol. 62, no. 5, pp. 1379-1384, 2005.

[20] M. Cengiz, A. K. Singh, and P. W. Grigsby, "Postoperative vaginal brachytherapy alone is the treatment of choice for grade 1-2, stage IC endometrial cancer," International Journal of Gynecological Cancer, vol. 15, no. 5, pp. 926-931, 2005.

[21] P. V. C. Rittenberg, R. J. Lotocki, M. S. Heywood, K. D. Jones, and G. V. Krepart, "High-risk surgical stage 1 endometrial cancer: Outcomes with vault brachytherapy alone," Gynecologic Oncology, vol. 89, no. 2, pp. 288-294, 2003.

[22] N. S. Horowitz, W. A. Peters, M. R. Smith et al., "Adjuvant high dose rate vaginal brachytherapy as treatment of stage I and II endometrial carcinoma," Obstetrics \& Gynecology, vol. 99, no. 2, pp. 235-240, 2002.

[23] Á. Rovirosa, A. Herreros, C. Camacho et al., "Comparative results of three short brachytherapy schedules as exclusive treatment in postoperative endometrial carcinoma," Brachytherapy, 2017.

[24] Á. Rovirosa, C. Ascaso, A. Herreros et al., "A new short daily brachytherapy schedule in postoperative endometrial carcinoma. Preliminary results," Brachytherapy, vol. 16, no. 1, pp. 147-152, 2017.

[25] K. Townamchai, L. Lee, and A. N. Viswanathan, "A novel low dose fractionation regimen for adjuvant vaginal brachytherapy in early stage endometrioid endometrial cancer," Gynecologic Oncology, vol. 127, no. 2, pp. 351-355, 2012.

[26] J. Straughn, W. K. Huh, F. Kelly et al., "Conservative management of Stage I endometrial carcinoma after surgical staging," Gynecologic Oncology, vol. 84, no. 2, pp. 194-200, 2002.

[27] E. F. Dunn, H. Geye, C. S. Platta et al., "Predictive factors of recurrence following adjuvant vaginal cuff brachytherapy alone 
for stage i endometrial cancer," Gynecologic Oncology, vol. 133, no. 3, pp. 494-498, 2014.

[28] P. Aristizabal, O. Graesslin, E. Barranger et al., "A suggested modification to FIGO stage i endometrial cancer," Gynecologic Oncology, vol. 133, no. 2, pp. 192-196, 2014.

[29] M. M. Alhilli, J. N. Bakkum-Gamez, A. Mariani et al., "Riskadjusted outcomes in elderly endometrial cancer patients: Implications of the contrasting impact of age on progressionfree and cause-specific survival," Gynecologic Oncology, vol. 138, no. 1, pp. 133-140, 2015.

[30] M. Arenas, M. Gascón, À. Rovirosa et al., "The effect of lymphadenectomy and radiotherapy on recurrence and survival in endometrial carcinoma. Experience in a population reference centre," Reports of Practical Oncology and Radiotherapy, vol. 20, no. 1, pp. 50-56, 2015.

[31] M. M. Alhilli, K. C. Podratz, S. C. Dowdy et al., "Preoperative biopsy and intraoperative tumor diameter predict lymph node dissemination in endometrial cancer," Gynecologic Oncology, vol. 128, no. 2, pp. 294-299, 2013.

[32] N. T. Kizer, F. Gao, S. Guntupalli et al., "Lower uterine segment involvement is associated with poor outcomes in earlystage endometrioid endometrial carcinoma," Annals of Surgical Oncology, vol. 18, no. 5, pp. 1419-1424, 2011.

[33] S. Chattopadhyay, P. Cross, A. Nayar, K. Galaal, and R. Naik, "Tumor size: A better independent predictor of distant failure and death than depth of myometrial invasion in international federation of gynecology and obstetrics stage I endometrioid endometrial cancer," International Journal of Gynecological Cancer, vol. 23, no. 4, pp. 690-697, 2013.

[34] O. Lavie, L. Uriev, M. Gdalevich et al., "The outcome of patients with stage I endometrial cancer involving the lower uterine segment," International Journal of Gynecological Cancer, vol. 18, no. 5, pp. 1079-1083, 2008.

[35] G. Canlorbe, S. Bendifallah, E. Laas et al., "Tumor Size, an Additional Prognostic Factor to Include in Low-Risk Endometrial Cancer: Results of a French Multicenter Study," Annals of Surgical Oncology, vol. 23, no. 1, pp. 171-177, 2016. 


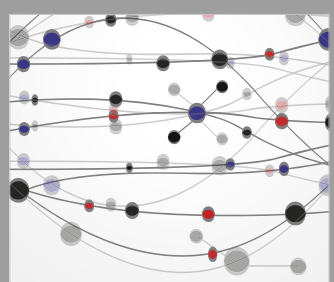

The Scientific World Journal
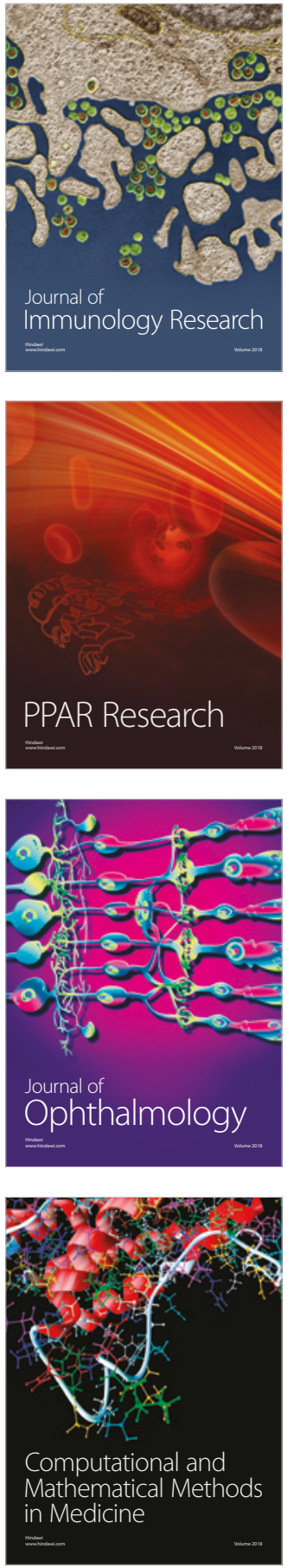

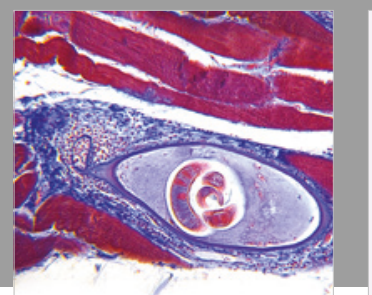

Gastroenterology Research and Practice

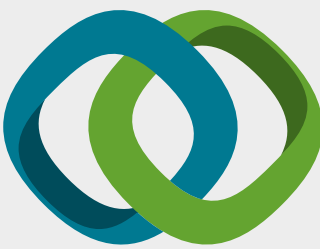

\section{Hindawi}

Submit your manuscripts at

www.hindawi.com
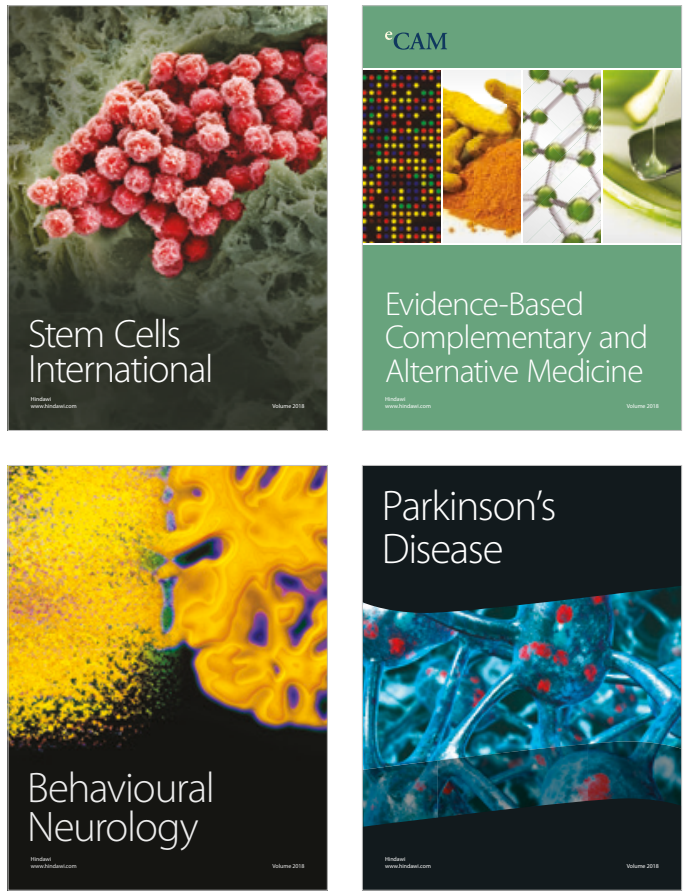

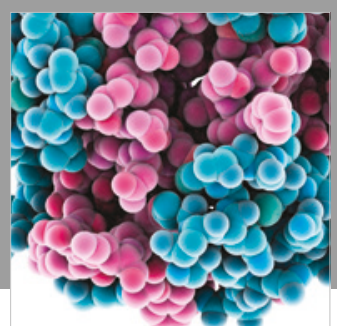

ournal of

Diabetes Research

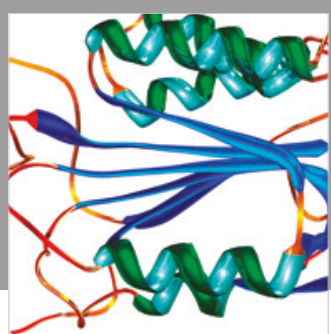

Disease Markers
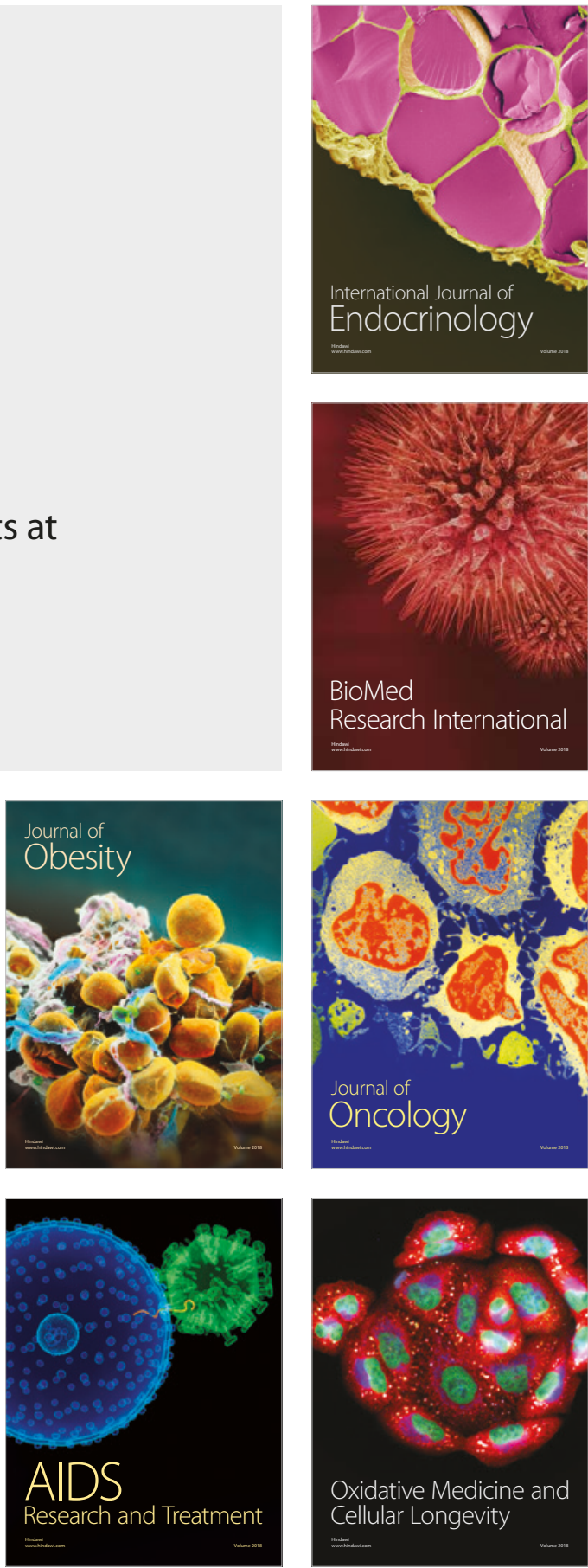